\title{
Fine-Tuning the Expression of Duplicate Genes by Translational Regulation in Arabidopsis and Maize
}

\section{OPEN ACCESS}

Edited by:

Michael Eric Schranz,

Wageningen University \& Research,

Netherlands

Reviewed by:

Mingsheng Chen,

Institute of Genetics and Developmental Biology (CAS),

China

Guixia Xu,

Institute of Botany (CAS), China

*Correspondence:

Sishuo Wang

sishuowang@hotmail.ca

Youhua Chen

chenyh@cib.ac.cn

Specialty section:

This article was submitted to Plant Systematics and Evolution,

a section of the journal

Frontiers in Plant Science

Received: 04 February 2019

Accepted: 05 April 2019

Published: 08 May 2019

Citation:

Wang S and Chen Y (2019)

Fine-Tuning the Expression of Duplicate Genes by Translational Regulation in Arabidopsis and Maize.

Front. Plant Sci. 10:534.

doi: 10.3389/fp/s.2019.00534

\section{Sishuo Wang ${ }^{1,2,3 *}$ and Youhua Chen ${ }^{1 *}$}

${ }^{1}$ Chengdu Institute of Biology, Chinese Academy of Sciences, Chengdu, China, ${ }^{2}$ Department of Botany, Faculty of Science, The University of British Columbia, Vancouver, BC, Canada, ${ }^{3}$ School of Life Sciences, The Chinese University of Hong Kong, Sha Tin, Hong Kong

Plant genomes are extensively shaped by various types of gene duplication. However, in this active area of investigation, the vast majority of studies focus on the sequence and transcription of duplicate genes, leaving open the question of how translational regulation impacts the expression and evolution of duplicate genes. We explored this issue by analyzing the ribo- and mRNA-seq data sets across six tissue types and stress conditions in Arabidopsis thaliana and maize (Zea mays). We dissected the relative contributions of transcriptional and translational regulation to the divergence in the abundance of ribosome footprint (RF) for different types of duplicate genes. We found that the divergence in RF abundance was largely programmed at the transcription level and that translational regulation plays more of a modulatory role. Intriguingly, translational regulation is characterized by its strong directionality, with the divergence in translational efficiency (TE) globally counteracting the divergence in mRNA abundance, indicating partial buffering of the transcriptional divergence between paralogs by translational regulation. Divergence in TE was associated with several sequence features. The faster-evolving copy in a duplicate pair was more likely to show lower RF abundance, which possibly results from relaxed purifying selection compared with its paralog. A considerable proportion of duplicates displayed differential TE across tissue types and stress conditions, most of which were enriched in photosynthesis, energy production, and translation-related processes. Additionally, we constructed a database TDPDG-DB (http://www.plantdupribo.tk), providing an online platform for data exploration. Overall, our study illustrates the roles of translational regulation in fine-tuning duplicate gene expression in plants.

Keywords: gene duplication, genome evolution, plant genomics, translational regulation, expression evolution

\section{INTRODUCTION}

One of the most prominent genomic differences between plants and other eukaryotes is the prevalence of duplicate genes in plant genomes (Panchy et al., 2016). Phylogenomic analyses have provided mounting evidence for recurrent episodes of ancient whole-genome duplication (WGD) throughout the evolutionary history of plants, with each WGD event superimposed on the genomic

Abbreviations: GO, Gene Ontology; $K_{\mathrm{a}}$, non-synonymous substitution rate; $K_{\mathrm{s}}$, synonymous substitution rate; RF, ribosome footprint; TE, translational efficiency; WGD, whole-genome duplication. 
remnants of more ancient ones (Bowers et al., 2003; Adams and Wendel, 2005; Van De Peer et al., 2009; Jiao et al., 2011; Ruprecht et al., 2017; Ren et al., 2018; Reuscher et al., 2018). In addition, duplicate genes can be generated from other mechanisms (e.g., tandem duplication), which, together with WGD, contribute to the great preponderance of duplicate genes in plant genomes (Panchy et al., 2016; Wang et al., 2018). Gene duplication provides an enormous reservoir of new genes for the innovation of functions and phenotypic traits, and is a primary force in driving genome evolution of flowering plants (Flagel and Wendel, 2009; Schranz et al., 2012; Dai et al., 2014; Soltis and Soltis, 2016; Jiao, 2018).

Investigating gene duplication and the evolutionary fates of genes after duplication is of fundamental importance in the understanding of plant genomes. One of the most important aspects of this issue is to understand expression conservation and divergence between paralogs. Several models have been proposed to interpret this issue (Zhang, 2003). Paralogs may subdivide their ancestral functions such that both copies become essential and are selectively retained (subfunctionalization) (Hughes, 1994; Force et al., 1999). Alternatively, one duplicate copy might evolve novel expression patterns or functions (neofunctionalization) (Ohno, 1970; Hughes et al., 2014). Counterbalancing these ideas is the gene dosage hypothesis, where both paralogs are subjected to constraints on dosage balance and show high conservation in sequence and expression (Edger and Pires, 2009; Conant et al., 2014). These evolutionary scenarios are not mutually exclusive [e.g., constraints on gene dosage may provide long enough time for duplicates to diverge in function (Force et al., 1999; Conant et al., 2014; Vaattovaara et al., 2019)], but their relative contributions remain to be explored in depth.

To date, our understanding of the expression between duplicate genes has been largely guided by studies using transcriptomic data. By microarray and RNA-seq analysis, many studies have demonstrated the divergence between paralogs in transcription (Li et al., 2005; Ganko et al., 2007; Roulin et al., 2013). However, gene expression is a dynamic process including transcription, translation, and protein turnover; transcript abundance may not always be biologically meaningful (Vogel and Marcotte, 2012; Bailey-Serres, 2013; Shah et al., 2013; Gamm et al., 2014; Liu et al., 2016). For example, several studies have estimated the divergence in microRNA regulation between paralogs in various plant species, and suggested its contributions to expression divergence between duplicates (Guo et al., 2008; Sun et al., 2015; Wang and Adams, 2015). It was also shown that more than $85 \%$ of paralogs in Arabidopsis show divergence in nonsense-mediated decay induced by alternative splicing (Tack et al., 2014). These studies reveal the crucial roles of posttranscriptional regulation between paralogous genes.

Translation is one of the most energy-consuming processes in cell (Buttgereit and Brand, 1995; Lynch and Marinov, 2015). As such, fine regulation of translation is very important in regulating the level of gene expression and protein synthesis to the actual needs. Translational regulation might particularly be important for plants considering their complex translational apparatus and additional genetic systems in chloroplasts and mitochondria (Ferrando et al., 2017). However, for a long period, our understanding of translational regulation is limited to a small number of genes, and the link between transcript abundance and protein synthesis still waits to be understood (Koh et al., 2012; Hu et al., 2013). Recently, ribosome profiling technology (riboseq) (Ingolia et al., 2009) has emerged as a powerful method to identify translating mRNAs, which provides an alternative and robust way to assess gene expression and allows for identification of translational regulation on a genome-wide scale (Ingolia, 2014; Merchante et al., 2017). Using ribo-seq and its related technologies, several studies have revealed the important roles of translational regulation of gene expression in plants, suggesting that the functional patterns of the expression of many genes may not be established until translation (Juntawong et al., 2014; Lei et al., 2015; Hsu et al., 2016; Bai et al., 2017). Coate et al. (2014) analyzed the translatome of a recently formed polyploid Glycine. This study revealed rapid changes in translation shortly after polyploidization, suggesting a previously unappreciated role of translational regulation in reducing expression differences between polyploid and parents. Although the results of this study are very encouraging, it is worth noting that the vast majority of modern plants are diploidized paleopolyploids (Panchy et al., 2016; Pont and Salse, 2017). Mechanisms of translational regulation in recently formed polyploids might not apply to other modern plants where on average $65 \%$ of genes in the genome are duplicates that have been retained for millions of years (Panchy et al., 2016). Considering the importance of translation in gene expression and the prevalence of duplicates in plant genomes, a thorough study of translational regulation between paralogs in plants is urgently needed.

To investigate translational divergence between paralogs and its impacts on duplicate gene evolution, we carried out a comprehensive analysis of translational regulation of paralogs derived from WGD as well as tandem duplication in Arabidopsis thaliana and maize (Zea mays) by integrating six paired ribo-/mRNA-seq data sets from different tissue types and stress conditions. We analyzed the divergence between the RF abundance (i.e., the abundance of ribosome-associated reads mapped in ribo-seq) and mRNA abundance for duplicate genes. We found that while the divergence in RF abundance is mainly underlined at the transcriptional level, translational regulation tends to buffer transcriptional divergence between paralogs. In addition, we explored tissue- and stress-specific translational regulation between paralogs. We also analyzed the relationship between translational regulation and evolutionary rate for duplicates. Finally, we present on online database TDPDG-DB ${ }^{1}$ for data exploration.

\section{MATERIALS AND METHODS}

\section{Selection of Duplicate Gene Pairs}

We obtained the 3,183 pairs of Arabidopsis duplicates derived from the alpha WGD identified in Bowers et al. (2003). Maize WGD duplicates and their information regarding subgenome were retrieved from Schnable et al. (2011). Tandem duplicates were selected using the following procedure (Zou et al., 2009;

\footnotetext{
${ }^{1}$ http://plantdupribo.tk
} 
Liu et al., 2011). First, we clustered duplicate genes into tandem clusters if they (i) belong to the same family, (ii) are separated by at most 10 genes, and (iii) are located within $100 \mathrm{~kb}$ on the same chromosome. Then, for tandem gene clusters with more than two members, two genes were randomly chosen as the representative duplicate genes of the cluster. In total, a set of 2002 and 1706 pairs of tandem duplicates were collected for Arabidopsis and maize, respectively. The rest genes were classified as other types of duplicates, if they had non-self BLASTP hits with E-value lower than or equal to $1 \mathrm{e}-10$, or singletons, if they had no non-self BLASTP hits with E-values less than or equal to $1 \mathrm{e}-3$ (Wang and Adams, 2015). For genes with multiple isoforms, only the one with the longest sequence was selected as the representative. Sequence format conversion and processing were conducted with BEDOPS v2.4.14 (Neph et al., 2012), and custom scripts written in Ruby (Goto et al., 2010).

\section{mRNA-Seq and Ribo-Seq Data Sets and Read Mapping}

We collected deep-sequencing mRNA- and ribo-seq data sets of root and shoot from Hsu et al. (2016) and seedlings under normal and sublethal hypoxia stress conditions from Juntawong et al. (2014) for A. thaliana (Supplementary Table S1). We retrieved mRNA- and ribo-seq data of maize seedling under normal and drought conditions from Lei et al. (2015). mRNA- and riboseq data sets of the same tissue type or stress condition were generated from the same study. All of these data sets have at least two biological replicates.

Reads shorter than 20 nucleotides were removed before mapped to the genome. Terminal nucleotides with the sequencing quality less than or equal to 20 were trimmed by Cutadapt v1.3 (Martin, 2011). Reads were mapped to the reference genome with STAR v2.4.2a (Dobin et al., 2013) with the parameters "STAR -genomeDir index -readFilesIn fastqs outSAMtype BAM SortedByCoordinate -alignIntronMax 25000 -outSAMstrandField intronMotif”.

\section{Calculation of the Relative Divergence of RF and mRNA Abundance}

Fragments per kilobase per million mapped fragments (FPKM) for each gene was calculated with Cufflinks v2.2.1 (Trapnell et al., 2014). Since all of the ribo- and mRNA-seq data sets used in this study have multiple replicates, the expression level of each gene was averaged over all replicates in subsequent analysis. Because very lowly expressed genes are likely to be artifacts (Bhargava et al., 2014), we filtered out genes with average FPKM lower than 0.1 (Chettoor et al., 2014; Joag et al., 2016; Sun et al., 2018).

The signed relative divergence of RF or mRNA abundance is calculated as $(\mathrm{X} 2-\mathrm{X} 1) /(\mathrm{X} 1+\mathrm{X} 2)$, where $\mathrm{X} 1$ and $\mathrm{X} 2$ represent the FPKM value in the ribo- or mRNA-seq of the first and the second gene in a pair of duplicates, respectively, as calculated for the relative divergence of sequence evolutionary rate shown above. This measure quantifies the relative RF or mRNA abundance difference between a pair of paralogs by normalizing the overall RF or mRNA abundance of the pair (Conant and Wagner, 2003; Kim and Yi, 2006; Keller and Yi, 2014).

\section{Calculation of Translational Efficiency}

TE was calculated as ribo-seq FPKM/mRNA-seq FPKM as previously described (Ingolia et al., 2009), and has been used as a proxy of the translational speed and accuracy in many studies (Gerashchenko et al., 2012; Juntawong et al., 2014; Lei et al., 2015; Wang et al., 2015). The relative divergence of TE was calculated as $(\mathrm{X} 2-\mathrm{X} 1) /(\mathrm{X} 1+\mathrm{X} 2)$, where $\mathrm{X} 1$ and $\mathrm{X} 2$ represent the $\mathrm{TE}$ of the first and the second paralog, respectively.

Differentially translated genes across tissue types or stress conditions were identified using RiboDiff, which utilizes generalized linear model strategies to detect genes showing differential TE between data sets (Zhong et al., 2017). To perform analysis with RiboDiff, uniquely mapped reads from each mRNAseq and ribo-seq dataset were counted for each gene using featureCounts implemented in subread v1.4.6 (Liao et al., 2013). Genes with FDR-adjusted (Benjamini and Hochberg's method) $P$-value lower than 0.05 were flagged as genes that display differential TE (Zhong et al., 2017).

\section{Calculation of Sequence Divergence and Identification of Asymmetric Evolution Between Duplicate Genes}

Calculation of the relative amino acid divergence and identification of asymmetric evolution between duplicate genes followed the procedure described in previous studies (Blanc and Wolfe, 2004; Liu et al., 2011). Protein sequences for each duplicate gene pair were aligned using MUSCLE v3.8.31 (Edgar, 2004), which was then used as an guide to generate codon alignment using PAL2NAL (Suyama et al., 2006). To identify orthologs, we collected protein sequences of Carica papaya and Vitis vinifera, as used in Liu et al. (2011), and added protein sequences from Theobroma cacao, Citrus sinensis, Fragaria vesca, Ricinus communis as the outgroup. For maize, Setaria italica, Sorghum bicolor, Oryza sativa, and Oropetium thomaeum were selected as outgroup species. These species were chosen as the outgroup since they split from the lineages to Arabidopsis or maize before the WGD event analyzed in this study, and do not show any evidence for WGD after their split (Ren et al., 2018).

We employed codeml implemented in PAML v4.7 (Yang, $2007)$ to determine the non-synonymous $\left(K_{\mathrm{a}}\right)$ and synonymous substitution $\left(K_{\mathrm{s}}\right)$ rate for all duplicate gene pairs. Triplets where the value of $K_{\mathrm{a}}$ between the paralogs in Arabidopsis (or maize) was larger than that between the paralogs in Arabidopsis (or maize) and the ortholog in the outgroup species were discarded (Blanc and Wolfe, 2004). The relative divergence of $K_{\mathrm{a}}\left(\right.$ or $\left.K_{\mathrm{a}} / K_{\mathrm{s}}\right)$ was defined as $(\mathrm{X} 2-\mathrm{X} 1) /(\mathrm{X} 1+\mathrm{X} 2)$, where $\mathrm{X} 1$ and $\mathrm{X} 2$ stand for the $K_{\mathrm{a}}$ (or $K_{\mathrm{a}} / K_{\mathrm{s}}$ ) for the two paralogs, respectively (Conant and Wagner, 2003; Fares et al., 2013). Then we computed the $\log$ likelihood $(\operatorname{lnL})$ of the triplets under two competing evolutionary models (Blanc and Wolfe, 2004; Liu et al., 2011). The first model assumes that evolutionary rates are unconstrained (i.e., asymmetric evolution), and the second model assumes that duplicate genes evolve at clock-like rates (i.e., symmetric evolution). To test whether the model of asymmetric evolution fits better than the model of symmetric evolution, we applied the likelihood ratio test (LRT). In brief, twice the difference of the log 
likelihood under the two models $[2 \Delta \ln \mathrm{L}$, where $\Delta \ln \mathrm{L}=\ln \mathrm{L}$ (no constraint) - $\ln L($ clock)] was compared against a chi-square distribution with one degree of freedom. Duplicate gene pairs with FDR-adjusted (Benjamini and Hochberg's method) $P$-values lower than 0.05 were determined to show asymmetrical protein sequence evolution.

\section{Gene Ontology Analysis}

Gene Ontology enrichment was analyzed using topGO implemented in the PlantRegMap platform (Jin et al., 2017). Those with FDR-adjusted $P$-value lower than 0.05 in the Fisher's exact test were considered as overrepresented GO categories.

\section{RNA Structure Prediction}

We used RNAfold, a core program from Vienna RNA v1.8.5 (Gruber et al., 2008) to predict the minimum free energy RNA secondary structure at default temperature $37^{\circ} \mathrm{C}$ with default parameters.

\section{RESULTS}

\section{Translational Buffering of Transcriptional Divergence Between Duplicates}

Translational regulation could act in two ways for duplicate genes, either in the same direction as transcriptional regulation to amplify the mRNA abundance divergence, or in the opposite direction to buffer the expression divergence established at the transcriptional level. To determine the directionality of translational regulation for duplicate genes, we calculated the signed relative divergence of $\mathrm{mRNA}$ abundance and TE as $(\mathrm{X} 2-\mathrm{X} 1) /(\mathrm{X} 1+\mathrm{X} 2)$, where $\mathrm{X} 1$ and $\mathrm{X} 2$ denote the mRNA abundance or TE for the two paralogs, respectively (see also section "Materials and Methods"). Defined as the amount of RF normalized to underlying mRNA abundance (Ingolia et al., 2009), TE is widely used as an indicator of the propensity of mRNA to undergo translation (see also section "Materials and Methods"). The relationship between the divergence of mRNA abundance and TE for WGD duplicates is depicted in Figure 1A. As shown in the figure, mRNA divergence displayed a strong correlation with TE divergence between WGD duplicates in both species regardless of tissue types and stress conditions (Figure 1A). Similarly, tandem duplicates exhibited significant negative correlation between mRNA abundance divergence and TE divergence in all analyzed data sets (Supplementary Figure S1A). The above results indicate that the duplicate copy with the higher mRNA abundance is more likely to display lower TE compared with its paralog.

To further illustrate this pattern, we calculated the proportions of duplicate genes where mRNA abundance divergence was reduced and increased at the translational level, respectively. The divergence in mRNA abundance for an average of 58 and 68\% of WGD duplicates in Arabidopsis and maize, respectively, was reduced when measured at the level of RF abundance, significantly higher than those with increased expression divergence when measured by RF abundance (Figure 1B).
Moreover, we restricted the analysis to duplicates that displayed at least twofold difference in TE. With 72 and $80 \%$ of WGD duplicates exhibiting reduced expression divergence in RF abundance in Arabidopsis and maize, respectively, the pattern was even stronger (Figure 1C). The similar pattern was found for tandem duplicates (Supplementary Figures S1B,C).

We further investigated sequence features that are potentially associated with TE divergence between paralogs. For both WGD and tandem duplicates in maize, the GC content of CDS was positively correlated with the relative divergence of TE, and the length and minimal free energy of the predicted secondary structure of $3^{\prime}$ UTR displayed negative correlation with TE divergence (Supplementary Tables S2, S3). In contrast, for Arabidopsis duplicates, the divergence of examined sequence features exhibited weak correlations with TE divergence (Supplementary Tables S2, S3). This suggests that multiple factors may act together on translational regulation and that the sequence features affecting TE divergence vary across species (Lei et al., 2015; Bai et al., 2017; Zhao et al., 2017).

\section{Expression Divergence Between Duplicates Is Mainly Underlined by Transcript Abundance}

Next, we dissected the relative contributions of transcriptional regulation and translational regulation to the divergence in RF abundance by comparing the fold differences of mRNA abundance and TE for each pair of paralogs (Gerashchenko et al., 2012). In all analyzed tissue types and stress conditions, the fold difference of mRNA abundance between paralogs was significantly greater than that of TE for both WGD duplicates (Figure 2A) and tandem duplicates (Supplementary Figure S2A). Specifically, in Arabidopsis, an average of $75 \%$ of WGD duplicates exhibited higher fold difference of mRNA abundance than that of TE (Figure 1B). The proportion stood at 65\%, on average, for maize WGD duplicates (Figure 1B). Similar patterns were found for tandem duplicates (Supplementary Figure S2). In addition, we did not detect significant difference in the proportion of duplicates with greater TE fold difference between WGD and tandem duplicates (Figure $\mathbf{2}$ and Supplementary Figure S2). The results indicate that the divergence in RF abundance was in general underlined by transcript abundance divergence for most duplicates in Arabidopsis and maize.

\section{The Faster-Evolving Paralog Copy Is More Likely to Exhibit a Lower RF Abundance}

To explore the association between expression divergence and sequence divergence for duplicate genes, we calculated the relative divergence in RF abundance and amino acid sequence between paralogs (see section "Materials and Methods" and Supplementary Table S4). We observed significant negative correlation of the relative divergence between RF abundance and amino acid sequence in all data sets except for maize tandem duplicates (Figure 3A and Supplementary Figure S3A). Further, 
A

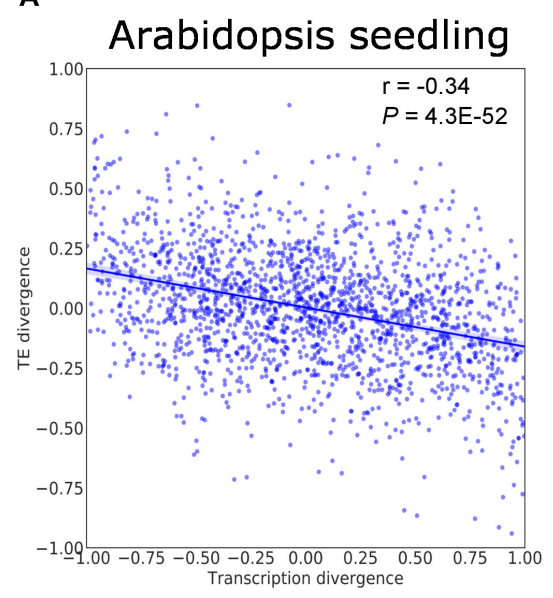

Arabidopsis shoot

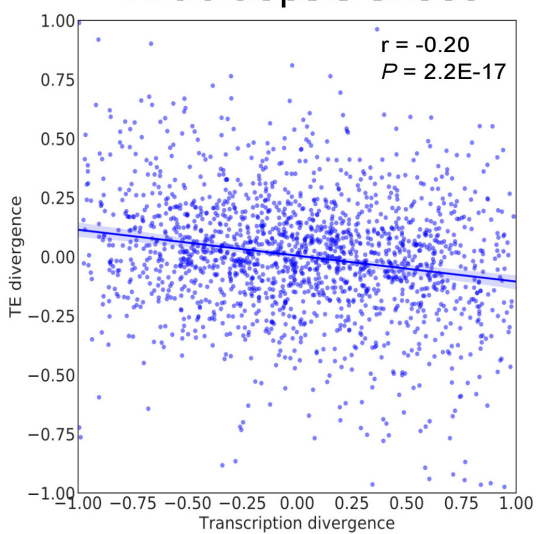

B

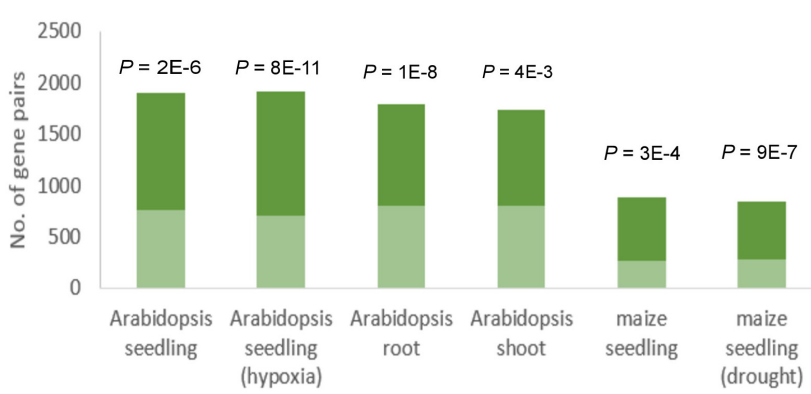

Arabidopsis seedling (hypoxia)

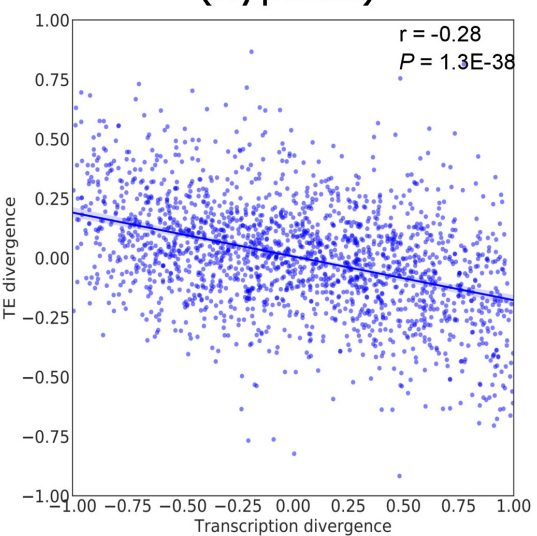

maize seedling

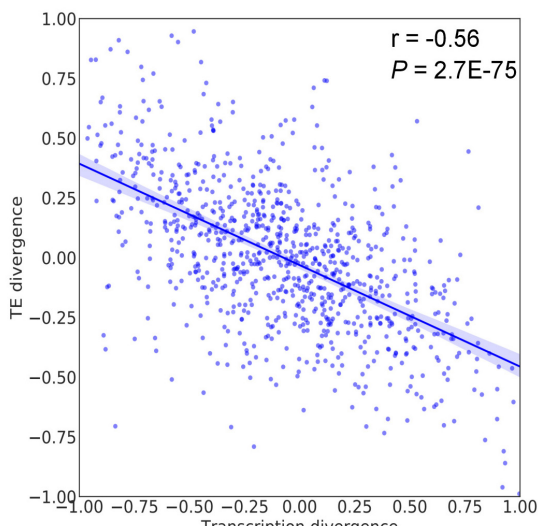

C

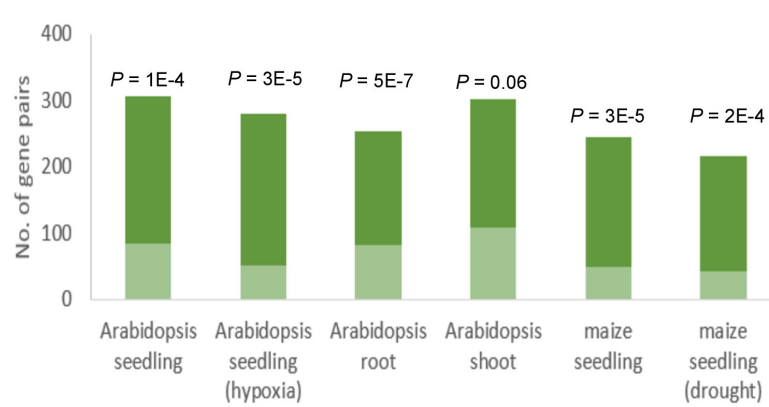

FIGURE 1 | Relationship between mRNA abundance divergence and TE divergence for WGD duplicates. (A) Scatter plots showing the correlation between the relative divergence in mRNA abundance and the relative divergence in TE. The Pearson's correlation coefficient and $P$-value are indicated. The shaded areas represent 95\% confidence interval in both plots. (B,C) Numbers of gene pairs whose transcriptional divergence decreases (referred to as "buffered") or increases (referred to as "reinforced") at the translational level for all WGD duplicates (B) and those with TE fold difference $\geq 2$ (C). P-values derived from the binomial test are shown above the bar.

we restricted the analysis to duplicates showing at least twofold difference in RF abundance and asymmetric sequence evolution (see section "Materials and Methods"), and the same pattern held true (Figure 3B and Supplementary Figure S3B). Similar to RF abundance divergence, mRNA abundance divergence was negatively correlated with protein sequence divergence
(Supplementary Tables S5, S6). However, we detected little correlation between TE divergence and amino acid divergence for duplicate genes (Supplementary Tables S5, S6). Thus, the fasterevolving copy was more likely to exhibit lower RF abundance than its slower-evolving paralog, which is mainly determined at the transcription level. 
A

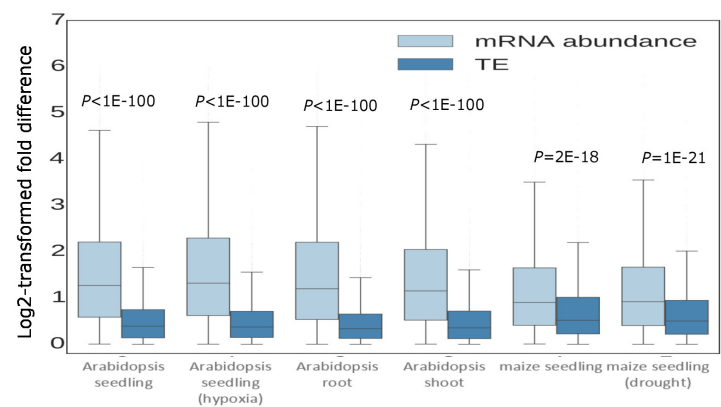

B

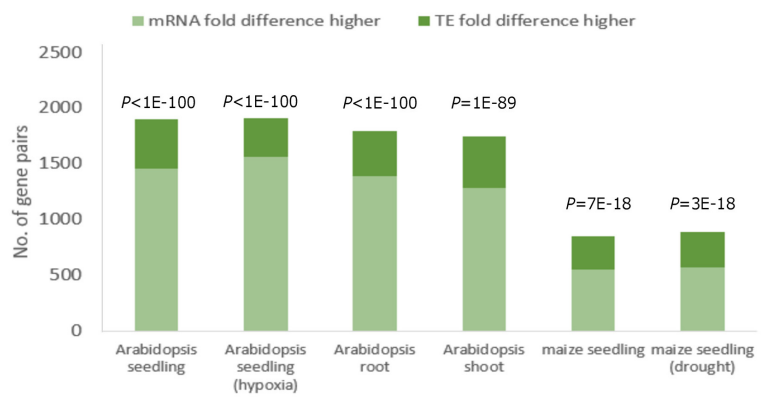

FIGURE 2 | Relative contributions of transcriptional and translational regulation to RF abundance divergence of WGD duplicates. (A) Log2-transformed fold differences in mRNA abundance and TE between WGD paralogs. Boxes extend from the first quartile (Q1) to the third quartile (Q3). The median is shown by a line inside the box. Whiskers extend to \pm 1.5 interquartile range (IQR). P-values derived from the Wilcoxon signed-rank test are shown. (B) Numbers of WGD duplicate pairs with higher fold difference in mRNA abundance than TE and with higher fold difference in TE than mRNA abundance. $P$-values derived from the binomial test are indicated.

We further calculated $K_{\mathrm{a}} / K_{\mathrm{s}}$ (the ratio of non-synonymous substitution rate and synonymous substitution rate) to examine the role of selection in the expression divergence between paralogs, as commonly used in prior studies (Jordan et al., 2004; Kondrashov, 2012; Hamaji et al., 2018). The $K_{\mathrm{a}} / K_{\mathrm{s}}$ divergence was negatively correlated with RF abundance divergence, although the correlation was in general weaker than that between amino acid divergence and RF abundance divergence (Supplementary Tables S5, S6). The $K_{\mathrm{a}} / K_{\mathrm{S}}$ of the vast majority of the paralog copy with the higher RF abundance ranged from $0.1-0.4$, and only a few displayed $K_{\mathrm{a}} / K_{\mathrm{s}}$ higher than 1.0 , which might be suggestive of positive selection (Supplementary Figure S4). This implies that the more lowly translated paralog tended to be under less selective constraints compared with its paralog.

\section{Tissue- and Stress-Specific Translational Regulation of Duplicates}

We then asked how translational regulation of duplicates varies across tissue types and in response to abiotic stress. We identified genes with differential TE across tissue types or stress conditions using RiboDiff between the normal and hypoxia condition for Arabidopsis seedling, between Arabidopsis root and shoot, and between the normal and drought condition for maize seedling (Supplementary Table S7; see section "Materials and Methods"). Genes with differential TE across tissue types or stress conditions were enriched in duplicate genes (i.e., WGD duplicates, tandem duplicates, and other types of duplicates; see section "Materials and Methods") compared with singletons (Figure 4A). This suggests that translational regulation preferentially regulates the expression of duplicates over singletons in plants. On average, 13 and 29\% of WGD duplicates had one copy differentially translated in Arabidopsis and maize, respectively (Figure 4B). For 2 and 10\% of WGD duplicates in Arabidopsis and maize, respectively, both copies showed differential TE between tissue types or stress conditions (Figure 4B). The higher proportion of duplicates with differentially translated genes in maize hints more translational regulation in maize than Arabidopsis, consistent with above results.
Gene ontology analysis revealed that the majority of duplicate genes with differential TE across tissues or stresses are those targeted to ribosome or chloroplast, and are involved in peptide biosynthesis, rRNA processing, photosynthesis, energy production, and protein degradation (Supplementary Table S8). As translation is one of the most energy-consuming processes in the cell (Buttgereit and Brand, 1995; Lynch and Marinov, 2015), the translational regulation of genes in these GO categories may serve as an energy conservation mechanism and help plants rapidly respond to environmental changes (Juntawong et al., 2014; Lei et al., 2015; Toribio et al., 2016; Wu et al., 2018). Of particular interest are genes encoding ribosomal proteins or proteins participating in ribosome biogenesis, because these genes are directly related to the translation of the entire transcriptome. This finding sparks the idea that, in response to stresses, plants may operate gene expression network by regulating the translation of translational apparatus, which in turn facilitates the translational regulation of the cell through a positive feedback (Juntawong et al., 2014). The partition of expression at the translational level adds a new layer of regulation for duplicate genes, which might facilitate functional divergence and long-term retention of both paralogs.

\section{TDPDG-DB: An Online Database for Translational Divergence of Plant Duplicate Genes}

To compile an archive of the translational regulation of plant duplicate genes and facilitate their research, we developed an online database TDPDG-DB (Translational Divergence of Plant Duplicate Genes Database ${ }^{2}$ ), which comprises all analyzed data sets in this study and makes them easily accessible to researchers (Supplementary Figure S5A). Through the "Search" interface, researchers can easily search for genes of interest by the data set, type of gene duplication, gene locus name, the fold difference of RF abundance and TE level, or any combination of the above (Supplementary Figure S5B). Users can further view the

\footnotetext{
${ }^{2} \mathrm{http}: / /$ plantdupribo.tk
} 
A

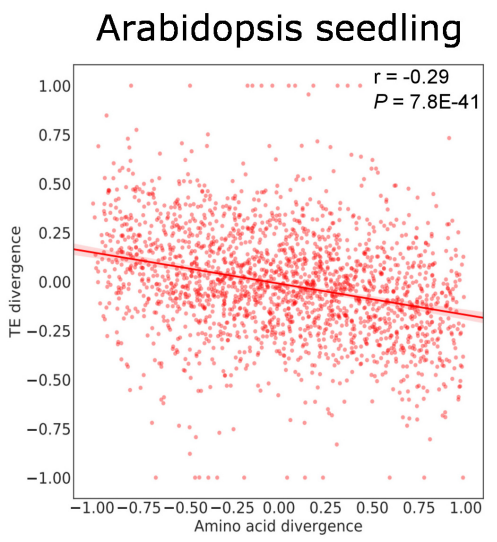

Arabidopsis shoot

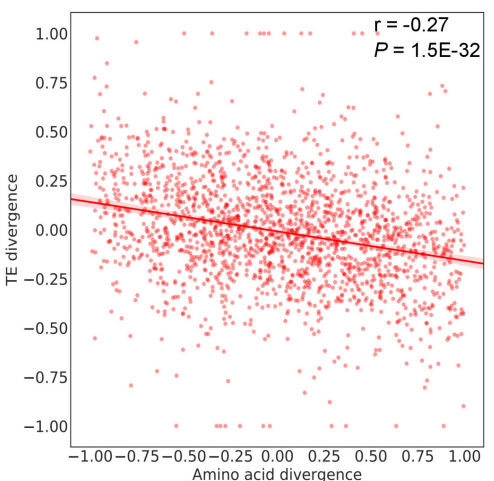

Arabidopsis seedling

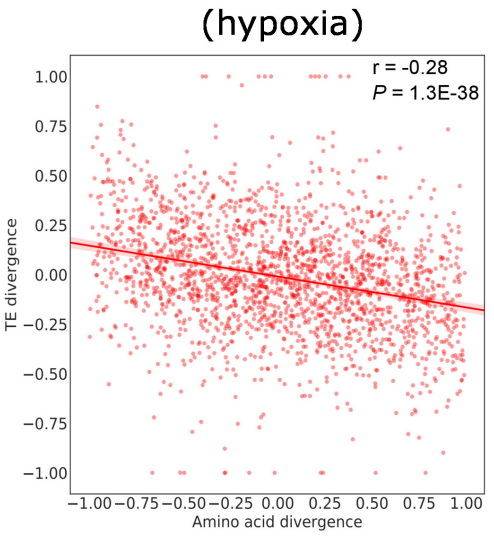

Amino acid divergence

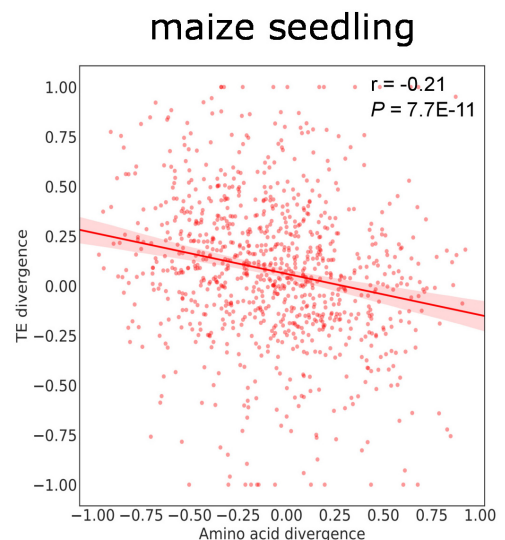

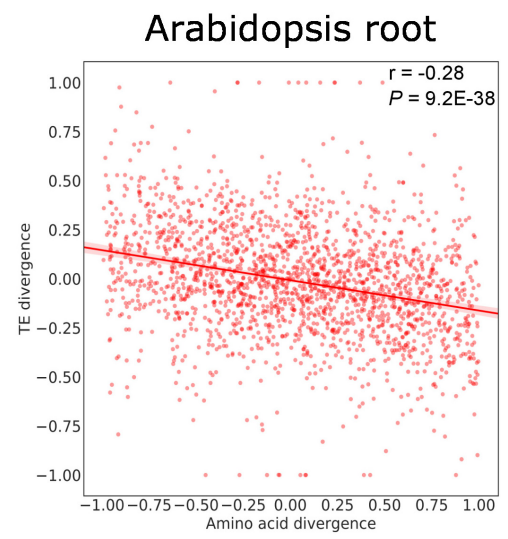

maize seedling (drought)

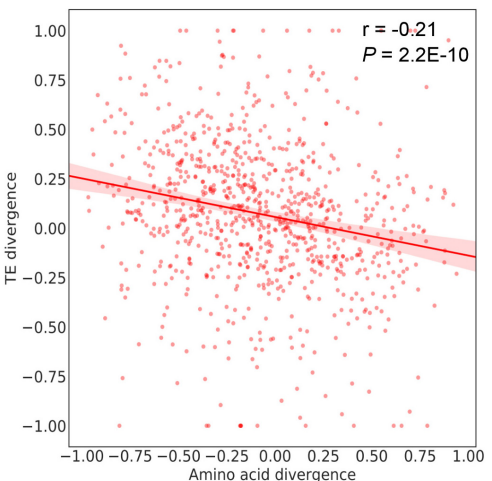

B n slower-evolving घ faster-evolving

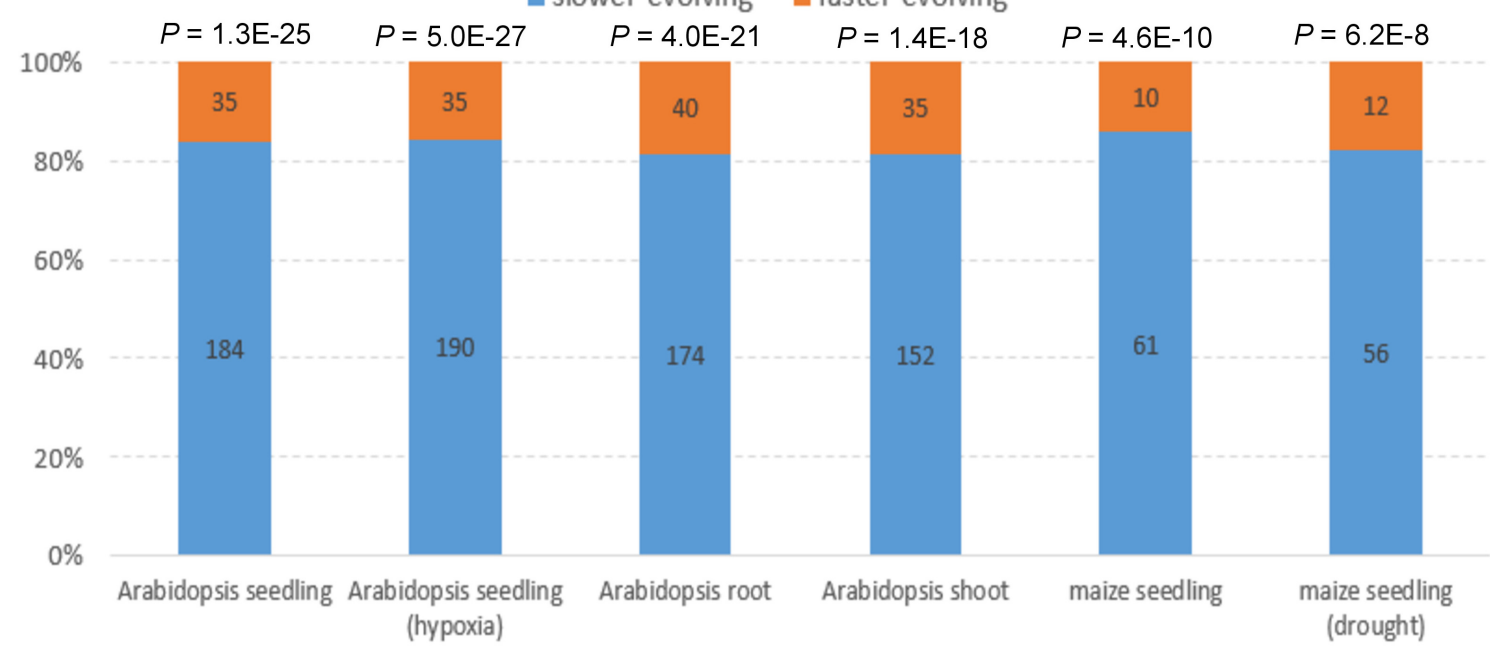

FIGURE 3 | Relationship between RF abundance divergence and amino acid divergence for WGD duplicates. Amino acid divergence is measured as non-synonymous substitution rate $\left(K_{\mathrm{a}}\right.$ ), as widely used in prior studies (Li et al., 2005; Ganko et al., 2007; Hakes et al., 2007). (A) Correlation between the RF abundance divergence and amino acid sequence divergence. The Pearson's correlation coefficient and $P$-value are indicated. The shaded areas represent $95 \%$ confidence interval in both plots. (B) Proportion of the paralog copy with the higher RF abundance between the slower- and faster-evolving copy in a duplicate pair. The $y$-axis represents the proportion of the paralog with the higher RF abundance. Bars in blue and yellow denote the slower-evolving and faster-evolving paralog copy, respectively. Numbers of duplicate pairs are given on the bar. $P$-values derived from a binomial test are shown above the bar. 


\section{A}

\section{singletons duplicates}
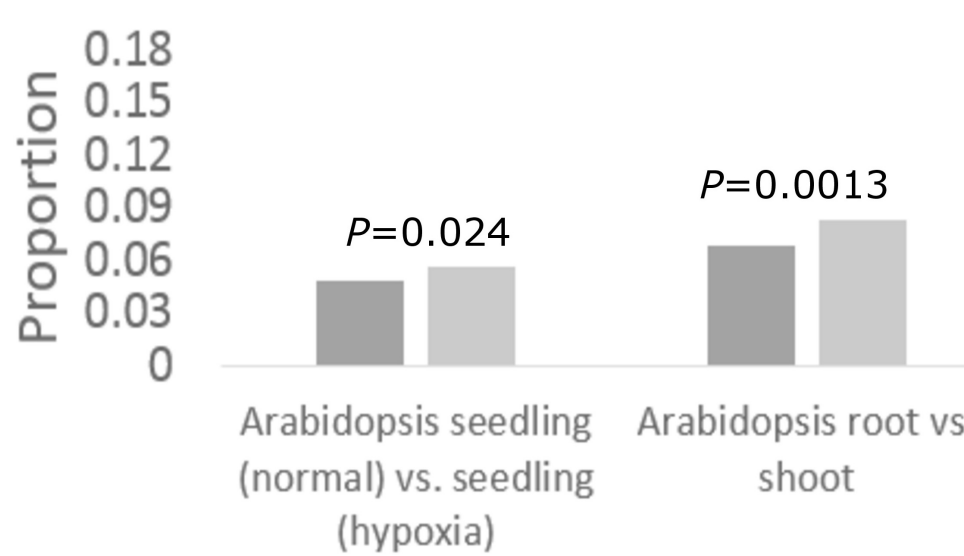

$P<2.2 \mathrm{E}-16$

0.18

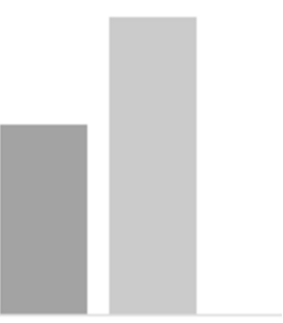

(normal) vs. seedling

(hypoxia)

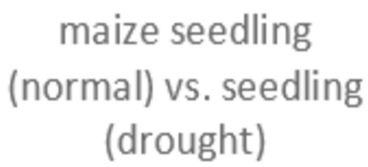

B - neither $\quad$ either $\quad$ both

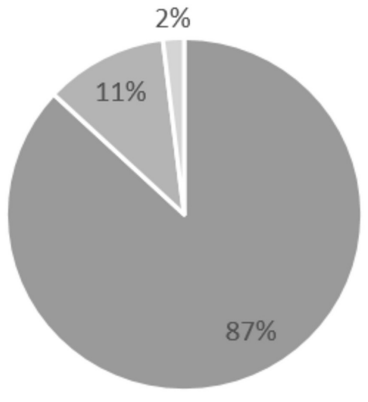

Arabidopsis seedling (normal) vs. seedling (hypoxia)

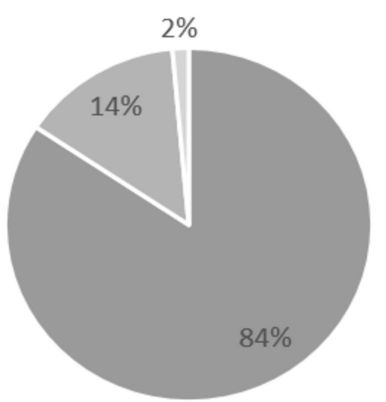

Arabidopsis root vs. shoot

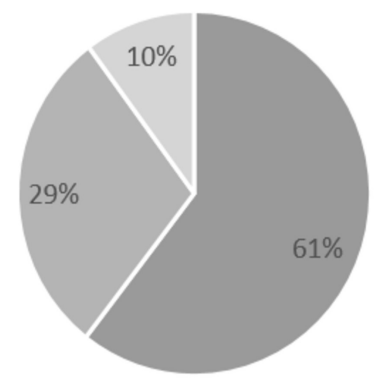

maize seedling (normal) vs. seedling (drought)

FIGURE 4 | Tissue- and stress-specific translational regulation of WGD duplicates. (A) Proportions of duplicates and singletons that showed differential TE between tissue types or under stress conditions. P-values obtained from the chi-squared test are shown above the bar. (B) Pie charts showing the proportion of WGD duplicates where neither copy (referred to as "neither"), one copy (referred to as "either"), and both copies (referred to as "both") showed differential TE.

details of the translational divergence as well as other related information of each paralog pair by clicking on the gene name in the search results (Supplementary Figure S5C). Additionally, users can download the original data deposited in the database by clicking on "Download" in the main toolbar.

\section{DISCUSSION}

Gene expression is a complex stepwise process involving regulation at many layers. Although expression evolution between duplicate genes has been well studied at the transcriptional level, much less attention has been paid to translation, one of the most energy-consuming processes in the cell (Buttgereit and Brand, 1995; Lynch and Marinov, 2015). The negative correlation between the divergence of mRNA abundance and TE shown in our study reveals that the paralog copy with higher mRNA abundance tended to display lower TE. Therefore, translational regulation of duplicate genes more often counteracts than follows the divergence in mRNA abundance, which partially compensates for their divergence in mRNA abundance. Our current understanding of expression relies heavily on transcriptomic data (reviewed in Panchy et al., 2016). Coate et al. (2014) analyzed the translatome of a recently formed polyploid Glycine $(\sim 0.1 \mathrm{Mya})$, showing that changes in translation changed shortly after polyploidization to reduce expression differences between the polyploid and parents. This, together with our study, reveals the widespread impact of translational buffering on duplicate gene expression in Arabidopsis and maize, suggesting that the extent to which the expression and function of duplicate genes diverge is likely overestimated when measured only at the transcription level. As expression similarity is an important indicator of functional similarity (Blanc and Wolfe, 2004; Wagner, 2005), post-transcriptional buffering of expression divergence might reduce the functional 
divergence between paralogs. Though divergence in expression and function is often thought to be crucial to the retention of duplicate genes, functional redundancy to some extent between paralogs may also have its benefits. Studies have shown that redundancy between duplicate genes could lead to buffering effect against null mutations, thereby increasing the robustness of the gene regulatory network ( $\mathrm{Gu}$ et al., 2003; Dean et al., 2008; Diss et al., 2014; Keane et al., 2014). In addition, for some duplicate genes, especially those in the same complex or involved in the same pathways, it may be important to maintain the right dosage balance (Edger and Pires, 2009; Birchler and Veitia, 2012; Wang and Chen, 2018; Gout et al., 2019). In this regard, by buffering the expression divergence established at the transcriptional level, translational regulation might fine-tune the expression level of both paralog copies, and help them better maintain the appropriate gene dosage.

The idea of translational buffering can be illustrated by a pair of WGD-derived duplicates involved in signaling cascades, MAPKKK17 (AT2G32510) and MAPKKK18 (AT1G05100). While the two paralogs were shown to function redundantly in root, they displayed about fourfold transcriptional divergence (Danquah et al., 2015; $\mathrm{Li}$ et al., 2017). The present study suggests that the divergence was compensated by their different translational efficiencies ${ }^{3}$, which results in nearly no difference in RF abundance and might contribute to functional redundancy of the duplicates.

Note, however, that despite its global trend to buffer divergence in mRNA abundance, the impact of translational regulation for plant duplicate genes should not be overexaggerated. As clearly shown in the present study, the divergence in $\mathrm{TE}$ is of markedly smaller scale than the divergence of mRNA abundance between paralogs (Figure 2 and Supplementary Figure S2). The medians of the fold difference of mRNA abundance were roughly twice and 1.5 times the fold difference of RF abundance in Arabidopsis and maize, respectively (Figure 2 and Supplementary Figure S2). Additionally, despite the general pattern of translational buffering, for many paralogous genes, translational regulation likely led to more divergent expression between them (Figure $\mathbf{1}$ and Supplementary Figure S1). Hence, we argue that the expression divergence between paralogs is mainly set up at the transcription level, whereas translational regulation plays more of a modulatory role to fine-tune the expression of plant duplicate genes.

In addition, we showed that the paralog with the higher RF abundance in a duplicate pair tended to evolve more slowly and be under more selective constraints than the other copy. This is generally consistent with previous studies for duplicate genes (Pal et al., 2001; Kim and Yi, 2006) [see also Duret and Mouchiroud (2000); Nuzhdin et al. (2004), Subramanian and Kumar (2004); Zhang and He

${ }^{3}$ http://plantdupribo.tk/single_gene.php?pair=AT1G05100-AT2G32510\&tissue= root_Hsu_2016
(2005), Lemos et al. (2005), and Drummond et al. (2006) for genes that are not limited to duplicates]. The fast evolution might lead to changes in regulatory elements, which in turn triggers the decrease in expression (Arsovski et al., 2015). Alternatively, the reduction of expression could occur first, which relaxes the selection pressure against amino acid change and allows for the accelerated sequence evolution of the duplicate copy (Zhang, 2003). If selection on precise gene expression mainly acts on protein abundance, translational buffering might lead to more tolerance to the variation in transcription, as phenotypic effects in mRNA abundance variation between paralogs could be masked at the translational level (Castelo-Szekely et al., 2017). It is possible that the networks of transcriptional and translational regulation diverge by genetic compensation, such that mutations in translation might counteract the effects of mutations in transcription or vice versa, resulting in translational buffering of expression divergence between paralogs (Mcmanus et al., 2014). This could be achieved by differences in sequence features of UTR and CDS, as shown in this and previous studies (Lei et al., 2015; Hsu et al., 2016; Zhao et al., 2017).

Translational regulation of genes is starting to be appreciated, but is still poorly understood. To facilitate the research of translational regulation for plant duplicate genes, we developed an online database TDPDG- $\mathrm{DB}^{4}$. We hope that this database can serve as a useful platform for researchers in related fields. Future studies of molecular mechanisms of changes in TE will provide more insights into the translational divergence of duplicate genes. It would also be important to examine whether patterns found in this study hold true in other species, and apply comparative genomics to assess the evolutionary conservation of translational regulation across lineages.

\section{AUTHOR CONTRIBUTIONS}

SW and YC conceived the study. SW performed the analysis. SW and $\mathrm{YC}$ analyzed the data and wrote the manuscript.

\section{FUNDING}

This work was supported by the Hundred Talents Program, Chinese Academy of Sciences (Grant No. Y8C3041100).

\section{ACKNOWLEDGMENTS}

We thank Server4You for the support of the server. We appreciate the help of Mark Robinson in gene expression analysis. We also thank Keith Adams, a brilliant mentor who guided SW to the research of plant genome evolution.

\footnotetext{
${ }^{4} \mathrm{http}: / /$ plantdupribo.tk
} 


\section{SUPPLEMENTARY MATERIAL}

The Supplementary Material for this article can be found online at: https://www.frontiersin.org/articles/10.3389/fpls.2019.00534/ full\#supplementary-material

FIGURE S1 | Relationship between mRNA abundance divergence and TE divergence for tandem duplicates. (A) Scatter plots showing the correlation between the relative divergence in mRNA abundance and the relative divergence in TE. The Pearson's correlation coefficient and $P$-value are indicated. The shaded areas represent $95 \%$ confidence interval in both plots. (B,C) Numbers of gene pairs whose transcriptional divergence decreases (referred to as "buffered") or increases (referred to as "reinforced") at the translational level for all tandem duplicates $\mathbf{( B )}$ and those with TE fold difference $\geq 2$ (C). P-values derived from the binomial test are shown above the bar.

FIGURE S2 | Relative contributions of transcriptional and translational regulation to RF abundance divergence of tandem duplicates. (A) Log2-transformed fold differences in mRNA abundance and TE between paralogs. Boxes extend from the first quartile (Q1) to the third quartile (Q3). The median is shown by a line inside the box. Whiskers extend to \pm 1.5 interquartile range (IQR). $P$-values derived from the Wilcoxon signed-rank test are shown. (B) Numbers of tandem duplicate pairs with higher fold difference in mRNA abundance than TE and with higher fold difference in TE than mRNA abundance. $P$-values derived from the binomial test are indicated.

FIGURE S3 | Relationship between RF abundance divergence and amino acid divergence for tandem duplicates. (A) Correlation between the RF abundance divergence and amino acid sequence divergence. The Pearson's correlation coefficient and $P$-value are indicated. The shaded areas represent 95\% confidence interval in both plots. (B) Proportion of the paralog copy with the higher RF abundance between the slower- and faster-evolving copy in a duplicate

\section{REFERENCES}

Adams, K. L., and Wendel, J. F. (2005). Polyploidy and genome evolution in plants. Curr. Opin. Plant Biol. 8, 135-141.

Arsovski, A. A., Pradinuk, J., Guo, X. Q., Wang, S., and Adams, K. L. (2015). Evolution of Cis-regulatory elements and regulatory networks in duplicated genes of Arabidopsis. Plant Physiol. 169, 2982-2991. doi: 10.1104/pp.15.0 0717

Bai, B., Peviani, A., Van Der Horst, S., Gamm, M., Snel, B., Bentsink, L., et al. (2017). Extensive translational regulation during seed germination revealed by polysomal profiling. New Phytol. 214, 233-244. doi: 10.1111/nph.1 4355

Bailey-Serres, J. (2013). Microgenomics: genome-scale, cell-specific monitoring of multiple gene regulation tiers. Annu. Rev. Plant Biol. 64, 293-325. doi: 10.1146/ annurev-arplant-050312-120035

Bhargava, V., Head, S. R., Ordoukhanian, P., Mercola, M., and Subramaniam, S. (2014). Technical variations in low-input RNA-seq methodologies. Sci. Rep. 4:3678. doi: 10.1038/srep03678

Birchler, J. A., and Veitia, R. A. (2012). Gene balance hypothesis: connecting issues of dosage sensitivity across biological disciplines. Proc. Natl. Acad. Sci. U.S.A. 109, 14746-14753. doi: 10.1073/pnas.1207726109

Blanc, G., and Wolfe, K. H. (2004). Functional divergence of duplicated genes formed by polyploidy during Arabidopsis evolution. Plant Cell 16, 1679-1691. doi: $10.1105 /$ tpc.021410

Bowers, J. E., Chapman, B. A., Rong, J. K., and Paterson, A. H. (2003). Unravelling angiosperm genome evolution by phylogenetic analysis of chromosomal duplication events. Nature 422, 433-438. doi: 10.1038/nature01521

Buttgereit, F., and Brand, M. D. (1995). A hierarchy of Atp-consuming processes in mammalian-cells. Biochem. J. 312, 163-167. doi: 10.1042/bj3120163

Castelo-Szekely, V., Arpat, A. B., Janich, P., and Gatfield, D. (2017). Translational contributions to tissue specificity in rhythmic and constitutive gene expression. Genome Biol. 18:116. doi: 10.1186/s13059-017-1222-2 pair. The $y$-axis represents the proportion of the paralog with the higher RF abundance. Bars in blue and yellow denote the slower-evolving and faster-evolving paralog copy, respectively. Numbers of duplicate pairs are given on the bar. $P$-values derived from a binomial test are shown above the bar.

FIGURE S4 I Distribution of the value of $K_{\mathrm{a}} / K_{\mathrm{s}}$ of the paralog copy with the higher RF abundance for WGD (A) and tandem duplicates (B).

FIGURE S5 | The database for translational divergence of plant duplicate genes. (A) Homepage of the database. (B) The 'Search' interface of the database. (C) An examples of the search results.

TABLE S1 | Mapping information of all analyzed ribo-seq/mRNA-seq data sets.

TABLE S2 | Correlation between the relative divergence of TE and the relative divergence of sequence features in CDS and UTR for WGD duplicates.

TABLE S3 | Correlation between the relative divergence of TE and the relative divergence of sequence features in CDS and UTR for tandem duplicates.

TABLE S4 | Sources of genomics information of plants used in the study.

TABLE S5 | Correlation of the relative divergence between mRNA abundance, TE, and $K_{\mathrm{s}}$, and between RF abundance and $K_{\mathrm{a}} / K_{\mathrm{s}}$ for WGD duplicates. The Pearson's correlation coefficient and $P$-value are shown.

TABLE S6 | Correlation of the relative divergence between mRNA abundance, TE, and $K_{\mathrm{s}}$, and between RF abundance and $K_{\mathrm{a}} / K_{\mathrm{s}}$ for tandem duplicates. The Pearson's correlation coefficient and $P$-value are shown.

TABLE S7 | Identification of genes with differential TE across tissue types or stress conditions using RiboDiff.

TABLE S8 | Gene ontology (GO) categories enriched in duplicate genes with differential TE across tissue types or stress conditions.

Chettoor, A., Givan, S., Cole, R., Coker, C., Unger-Wallace, E., Vejlupkova, Z., et al. (2014). Discovery of novel transcripts and gametophytic functions via RNA-seq analysis of maize gametophytic transcriptomes. Genome Biol. 15:414. doi: 10.1186/s13059-014-0414-2

Coate, J. E., Bar, H., and Doyle, J. J. (2014). Extensive translational regulation of gene expression in an allopolyploid (Glycine dolichocarpa). Plant Cell 26, 136-150. doi: 10.1105/tpc.113.119966

Conant, G. C., Birchler, J. A., and Pires, J. C. (2014). Dosage, duplication, and diploidization: clarifying the interplay of multiple models for duplicate gene evolution over time. Curr. Opin. Plant Biol. 19, 91-98. doi: 10.1016/j.pbi.2014. 05.008

Conant, G. C., and Wagner, A. (2003). Asymmetric sequence divergence of duplicate genes. Genome Res. 13, 2052-2058. doi: 10.1101/gr.1252603

Dai, X. G., Hu, Q. J., Cai, Q. L., Feng, K., Ye, N., Tuskan, G. A., et al. (2014). The willow genome and divergent evolution from poplar after the common genome duplication. Cell Res. 24, 1274-1277. doi: 10.1038/cr.2014.83

Danquah, A., De Zelicourt, A., Boudsocq, M., Neubauer, J., Frey, N. F. D., Leonhardt, N., et al. (2015). Identification and characterization of an ABAactivated MAP kinase cascade in Arabidopsis thaliana. Plant J. 82, 232-244. doi: $10.1111 /$ tpj. 12808

Dean, E. J., Davis, J. C., Davis, R. W., and Petrov, D. A. (2008). Pervasive and persistent redundancy among duplicated genes in yeast. PLoS Genet. 4:e1000113. doi: 10.1371/journal.pgen.1000113

Diss, G., Ascencio, D., Deluna, A., and Landry, C. R. (2014). Molecular mechanisms of paralogous compensation and the robustness of cellular networks. J. Exp. Zool. Part B Mol. Dev. Evol. 322, 488-499. doi: 10.1002/jez.b.22555

Dobin, A., Davis, C. A., Schlesinger, F., Drenkow, J., Zaleski, C., Jha, S., et al. (2013). STAR: ultrafast universal RNA-seq aligner. Bioinformatics 29, 15-21. doi: 10.1093/bioinformatics/bts635

Drummond, D. A., Raval, A., and Wilke, C. O. (2006). A single determinant dominates the rate of yeast protein evolution. Mol. Biol. Evol. 23, 327-337. doi: $10.1093 / \mathrm{molbev} / \mathrm{msj} 038$ 
Duret, L., and Mouchiroud, D. (2000). Determinants of substitution rates in mammalian genes: expression pattern affects selection intensity but not mutation rate. Mol. Biol. Evol. 17, 68-74.

Edgar, R. C. (2004). MUSCLE: multiple sequence alignment with high accuracy and high throughput. Nucleic Acids Res. 32, 1792-1797. doi: 10.1093/nar/gkh340

Edger, P. P., and Pires, J. C. (2009). Gene and genome duplications: the impact of dosage-sensitivity on the fate of nuclear genes. Chromosome Res. 17, 699-717. doi: 10.1007/s10577-009-9055-9

Fares, M. A., Keane, O. M., Toft, C., Carretero-Paulet, L., and Jones, G. W. (2013). The roles of whole-genome and small-scale duplications in the functional specialization of Saccharomyces cerevisiae genes. PLoS Genet. 9:e1003176. doi: 10.1371/journal.pgen.1003176

Ferrando, A., Castellano, M. M., Lison, P., Leister, D., Stepanova, A. N., and Hanson, J. (2017). Editorial: relevance of translational regulation on plant growth and environmental responses. Front. Plant Sci. 8:2170. doi: 10.3389/fpls. 2017.02170

Flagel, L. E., and Wendel, J. F. (2009). Gene duplication and evolutionary novelty in plants. New Phytol. 183, 557-564. doi: 10.1111/j.1469-8137.2009.02923.x

Force, A., Lynch, M., Pickett, F. B., Amores, A., Yan, Y. L., and Postlethwait, J. (1999). Preservation of duplicate genes by complementary, degenerative mutations. Genetics 151, 1531-1545.

Gamm, M., Peviani, A., Honsel, A., Snel, B., Smeekens, S., and Hanson, J. (2014). Increased sucrose levels mediate selective mRNA translation in Arabidopsis. BMC Plant Biol. 14:306. doi: 10.1186/s12870-014-0306-3

Ganko, E. W., Meyers, B. C., and Vision, T. J. (2007). Divergence in expression between duplicated genes in Arabidopsis. Mol. Biol. Evol. 24, 2298-2309. doi: $10.1093 / \mathrm{molbev} / \mathrm{msm} 158$

Gerashchenko, M. V., Lobanov, A. V., and Gladyshev, V. N. (2012). Genomewide ribosome profiling reveals complex translational regulation in response to oxidative stress. Proc. Natl. Acad. Sci. U.S.A. 109, 17394-17399. doi: 10.1073/ pnas.1120799109

Goto, N., Prins, P., Nakao, M., Bonnal, R., Aerts, J., and Katayama, T. (2010). BioRuby: bioinformatics software for the Ruby programming language. Bioinformatics 26, 2617-2619. doi: 10.1093/bioinformatics/btq475

Gout, J.-F., Johri, P., Arnaiz, O., Doak, T. G., Bhullar, S., Couloux, A., et al. (2019). Universal trends of post-duplication evolution revealed by the genomes of 13 Paramecium species sharing an ancestral whole-genome duplication. bioRxiv [Preprint]. doi: 10.1101/573576

Gruber, A. R., Lorenz, R., Bernhart, S. H., Neuboock, R., and Hofacker, I. L. (2008). The Vienna RNA websuite. Nucleic Acids Res. 36, W70-W74. doi: 10.1093/nar/ gkn 188

Gu, Z. L., Steinmetz, L. M., Gu, X., Scharfe, C., Davis, R. W., and Li, W. H. (2003). Role of duplicate genes in genetic robustness against null mutations. Nature 421, 63-66. doi: 10.1038/nature01198

Guo, X. Y., Gui, Y. J., Wang, Y., Zhu, Q. H., Helliwell, C., and Fan, L. J. (2008). Selection and mutation on microRNA target sequences during rice evolution. BMC Genomics 9:454. doi: 10.1186/1471-2164-9-454

Hakes, L., Pinney, J. W., Lovell, S. C., Oliver, S. G., and Robertson, D. L. (2007). All duplicates are not equal: the difference between small-scale and genome duplication. Genome Biol. 8:R209.

Hamaji, T., Kawai-Toyooka, H., Uchimura, H., Suzuki, M., Noguchi, H., Minakuchi, Y., et al. (2018). Anisogamy evolved with a reduced sexdetermining region in volvocine green algae. Commun. Biol. 1:17. doi: 10.1038/ s42003-018-0019-5

Hsu, P. Y., Calviello, L., Wu, H. Y. L., Li, F. W., Rothfels, C. J., Ohler, U., et al. (2016). Super-resolution ribosome profiling reveals unannotated translation events in Arabidopsis. Proc. Natl. Acad. Sci. U.S.A. 113, E7126-E7135. doi: $10.1073 /$ pnas.1614788113

Hu, G. J., Koh, J., Yoo, M. J., Grupp, K., Chen, S. X., and Wendel, J. F. (2013). Proteomic profiling of developing cotton fibers from wild and domesticated Gossypium barbadense. New Phytol. 200, 570-582. doi: 10.1111/nph.12381

Hughes, A. L. (1994). The evolution of functionally novel proteins after gene duplication. Proc. Biol. Sci. 256, 119-124. doi: 10.1098/rspb.1994. 0058

Hughes, T. E., Langdale, J. A., and Kelly, S. (2014). The impact of widespread regulatory neofunctionalization on homeolog gene evolution following wholegenome duplication in maize. Genome Res. 24, 1348-1355. doi: 10.1101/gr. 172684.114
Ingolia, N. T. (2014). Ribosome profiling: new views of translation, from single codons to genome scale. Nat. Rev. Genet. 15, 205-213. doi: 10.1038/nrg3645

Ingolia, N. T., Ghaemmaghami, S., Newman, J. R. S., and Weissman, J. S. (2009). Genome-wide analysis in vivo of translation with nucleotide resolution using ribosome profiling. Science 324, 218-223. doi: 10.1126/science.1168978

Jiao, Y. N. (2018). Double the genome, double the fun: genome duplications in angiosperms. Mol. Plant 11, 357-358. doi: 10.1016/j.molp.2018.02.009

Jiao, Y. N., Wickett, N. J., Ayyampalayam, S., Chanderbali, A. S., Landherr, L., Ralph, P. E., et al. (2011). Ancestral polyploidy in seed plants and angiosperms. Nature 473, 97-100. doi: 10.1038/nature09916

Jin, J. P., Tian, F., Yang, D. C., Meng, Y. Q., Kong, L., Luo, J. C., et al. (2017). PlantTFDB 4.0: toward a central hub for transcription factors and regulatory interactions in plants. Nucleic Acids Res. 45, D1040-D1045. doi: 10.1093/nar/ gkw982

Joag, R., Stuglik, M., Konczal, M., Plesnar-Bielak, A., Skrzynecka, A., Babik, W., et al. (2016). Transcriptomics of intralocus sexual conflict: gene expression patterns in females change in response to selection on a male secondary sexual trait in the bulb mite. Genome Biol. Evol. 8, 2351-2357. doi: 10.1093/gbe/ evw169

Jordan, I. K., Wolf, Y. I., and Koonin, E. V. (2004). Duplicated genes evolve slower than singletons despite the initial rate increase. BMC Evol. Biol. 4:22. doi: 10.1186/1471-2148-4-22

Juntawong, P., Girke, T., Bazin, J., and Bailey-Serres, J. (2014). Translational dynamics revealed by genome-wide profiling of ribosome footprints in Arabidopsis. Proc. Natl. Acad. Sci. U.S.A. 111, E203-E212. doi: 10.1073/pnas. 1317811111

Keane, O. M., Toft, C., Carretero-Paulet, L., Jones, G. W., and Fares, M. A. (2014). Preservation of genetic and regulatory robustness in ancient gene duplicates of Saccharomyces cerevisiae. Genome Res. 24, 1830-1841. doi: 10.1101/gr.17679 2.114

Keller, T. E., and Yi, S. V. (2014). DNA methylation and evolution of duplicate genes. Proc. Natl. Acad. Sci. U.S.A. 111, 5932-5937. doi: 10.1073/pnas. 1321420111

Kim, S. H., and Yi, S. V. (2006). Correlated asymmetry of sequence and functional divergence between duplicate proteins of Saccharomyces cerevisiae. Mol. Biol. Evol. 23, 1068-1075. doi: 10.1093/molbev/msj115

Koh, J., Chen, S. X., Zhu, N., Yu, F. H., Soltis, P. S., and Soltis, D. E. (2012). Comparative proteomics of the recently and recurrently formed natural allopolyploid Tragopogon mirus (Asteraceae) and its parents. New Phytol. 196, 292-305. doi: 10.1111/j.1469-8137.2012.04251.x

Kondrashov, F. A. (2012). Gene duplication as a mechanism of genomic adaptation to a changing environment. Proc. Biol. Sci. 279, 5048-5057. doi: 10.1098/rspb. 2012.1108

Lei, L., Shi, J. P., Chen, J., Zhang, M., Sun, S. L., Xie, S. J., et al. (2015). Ribosome profiling reveals dynamic translational landscape in maize seedlings under drought stress. Plant J. 84, 1206-1218. doi: 10.1111/tpj.1 3073

Lemos, B., Bettencourt, B. R., Meiklejohn, C. D., and Hartl, D. L. (2005). Evolution of proteins and gene expression levels are coupled in Drosophila and are independently associated with mRNA abundance, protein length, and number of protein-protein interactions. Mol. Biol. Evol. 22, 1345-1354. doi: 10.1093/ molbev/msi122

Li, W. H., Yang, J., and Gu, X. (2005). Expression divergence between duplicate genes. Trends Genet. 21, 602-607. doi: 10.1016/j.tig.2005.08.006

Li, Y. Y., Cai, H. X., Liu, P., Wang, C. Y., Gao, H., Wu, C. G., et al. (2017). Arabidopsis MAPKKK18 positively regulates drought stress resistance via downstream MAPKK3. Biochem. Biophys. Res. Commun. 484, 292-297. doi: 10.1016/j.bbrc.2017.01.104

Liao, Y., Smyth, G. K., and Shi, W. (2013). The Subread aligner: fast, accurate and scalable read mapping by seed-and-vote. Nucleic Acids Res. 41:e108. doi: $10.1093 /$ nar/gkt214

Liu, S. L., Baute, G. J., and Adams, K. L. (2011). Organ and cell type-specific complementary expression patterns and regulatory neofunctionalization between duplicated genes in Arabidopsis thaliana. Genome Biol. Evol. 3, 1419-1436. doi: 10.1093/gbe/evr114

Liu, Y. S., Beyer, A., and Aebersold, R. (2016). On the dependency of cellular protein levels on mRNA abundance. Cell 165, 535-550. doi: 10.1016/j.cell.2016. 03.014 
Lynch, M., and Marinov, G. K. (2015). The bioenergetic costs of a gene. Proc. Natl. Acad. Sci. U.S.A. 112, 15690-15695. doi: 10.1073/pnas.1514974112

Martin, M. (2011). Cutadapt removes adapter sequences from high-throughput sequencing reads. EMBnetjournal 17, 10-12.

Mcmanus, C. J., May, G. E., Spealman, P., and Shteyman, A. (2014). Ribosome profiling reveals post-transcriptional buffering of divergent gene expression in yeast. Genome Res. 24, 422-430. doi: 10.1101/gr.164996.113

Merchante, C., Stepanova, A. N., and Alonso, J. M. (2017). Translation regulation in plants: an interesting past, an exciting present and a promising future. Plant J. 90, 628-653. doi: 10.1111/tpj.13520

Neph, S., Kuehn, M. S., Reynolds, A. P., Haugen, E., Thurman, R. E., Johnson, A. K., et al. (2012). BEDOPS: high-performance genomic feature operations. Bioinformatics 28, 1919-1920. doi: 10.1093/bioinformatics/bts277

Nuzhdin, S. V., Wayne, M. L., Harmon, K. L., and Mcintyre, L. M. (2004). Common pattern of evolution of gene expression level and protein sequence in Drosophila. Mol. Biol. Evol. 21, 1308-1317. doi: 10.1093/molbev/msh128

Ohno, S. (1970). Evolution by Gene Duplication. New York, NY: Springer-Verlag.

Pal, C., Papp, B., and Hurst, L. D. (2001). Highly expressed genes in yeast evolve slowly. Genetics 158, 927-931.

Panchy, N., Lehti-Shiu, M., and Shiu, S. H. (2016). Evolution of gene duplication in plants. Plant Physiol. 171, 2294-2316. doi: 10.1104/pp.16.00523

Pont, C., and Salse, J. (2017). Wheat paleohistory created asymmetrical genomic evolution. Curr. Opin. Plant Biol. 36, 29-37. doi: 10.1016/j.pbi.2017.01.001

Ren, R., Wang, H., Guo, C., Zhang, N., Zeng, L., Chen, Y., et al. (2018). Widespread whole genome duplications contribute to genome complexity and species diversity in angiosperms. Mol. Plant 11, 414-428. doi: 10.1016/j.molp.2018.0 1.002

Reuscher, S., Furuta, T., Bessho-Uehara, K., Cosi, M., Jena, K. K., Toyoda, A., et al. (2018). Assembling the genome of the African wild rice Oryza longistaminata by exploiting synteny in closely related Oryza species. Commun. Biol. 1:162. doi: 10.1038/s42003-018-0171-y

Roulin, A., Auer, P. L., Libault, M., Schlueter, J., Farmer, A., May, G., et al. (2013). The fate of duplicated genes in a polyploid plant genome. Plant J. 73, 143-153. doi: 10.1111/tpj.12026

Ruprecht, C., Lohaus, R., Vanneste, K., Mutwil, M., Nikoloski, Z., Van De Peer, Y., et al. (2017). Revisiting ancestral polyploidy in plants. Science Adv. 3:e1603195. doi: 10.1126/sciadv. 1603195

Schnable, J. C., Springer, N. M., and Freeling, M. (2011). Differentiation of the maize subgenomes by genome dominance and both ancient and ongoing gene loss. Proc. Natl. Acad. Sci. U. S. A. 108, 4069-4074. doi: 10.1073/pnas. 1101368108

Schranz, M. E., Mohammadin, S., and Edger, P. P. (2012). Ancient whole genome duplications, novelty and diversification: the WGD Radiation Lag-Time Model. Curr. Opin. Plant Biol. 15, 147-153. doi: 10.1016/j.pbi.2012.03.011

Shah, P., Ding, Y., Niemczyk, M., Kudla, G., and Plotkin, J. B. (2013). Rate-limiting steps in yeast protein translation. Cell 153, 1589-1601. doi: 10.1016/j.cell.2013. 05.049

Soltis, P. S., and Soltis, D. E. (2016). Ancient WGD events as drivers of key innovations in angiosperms. Curr. Opin. Plant Biol. 30, 159-165. doi: 10.1016/ j.pbi.2016.03.015

Subramanian, S., and Kumar, S. (2004). Gene expression intensity shapes evolutionary rates of the proteins encoded by the vertebrate genome. Genetics 168, 373-381. doi: 10.1534/genetics.104.028944

Sun, C., Wu, J., Liang, J. L., Schnable, J. C., Yang, W. C., Cheng, F., et al. (2015). Impacts of whole-genome triplication on MIRNA evolution in Brassica rapa. Genome Biol. Evol. 7, 3085-3096. doi: 10.1093/gbe/evv206

Sun, Y., Hou, H., Song, H. T., Lin, K., Zhang, Z. H., Hu, J. L., et al. (2018). The comparison of alternative splicing among the multiple tissues in cucumber. BMC Plant Biol. 18:5. doi: 10.1186/s12870-017-1217-x

Suyama, M., Torrents, D., and Bork, P. (2006). PAL2NAL: robust conversion of protein sequence alignments into the corresponding codon alignments. Nucleic Acids Res. 34, W609-W612.

Tack, D. C., Pitchers, W. R., and Adams, K. L. (2014). Transcriptome analysis indicates considerable divergence in alternative splicing between duplicated genes in Arabidopsis thaliana. Genetics 198, 1473-1481. doi: 10.1534/genetics. 114.169466
Toribio, R., Muñoz, A., Castro-Sanz, A. B., Ferrando, A., Berrocal-Lobo, M., and Castellan, M. M. (2016). "Evolutionary aspects of translation regulation during abiotic stress and development in plants," in Evolution of the Protein Synthesis Machinery and Its Regulation, eds G. Hernández and R. Jagus (Cham: Springer), 477-490. doi: 10.1007/978-3-319-39468-8_18

Trapnell, C., Roberts, A., Goff, L., Pertea, G., Kim, D., Kelley, D. R., et al. (2014). Differential gene and transcript expression analysis of RNA-seq experiments with TopHat and Cufflinks. Nat. Protoc. 7, 562-578. doi: 10.1038/nprot.201 2.016

Vaattovaara, A., Brandt, B., Rajaraman, S., Safronov, O., Veidenberg, A., Luklová, M., et al. (2019). Mechanistic insights into the evolution of DUF26-containing proteins in land plants. Commun. Biol. 2:56. doi: 10.1038/s42003-0190306-9

Van De Peer, Y., Fawcett, J. A., Proost, S., Sterck, L., and Vandepoele, K. (2009). The flowering world: a tale of duplications. Trends Plant Sci. 14, 680-688. doi: 10.1016/j.tplants.2009.09.001

Vogel, C., and Marcotte, E. M. (2012). Insights into the regulation of protein abundance from proteomic and transcriptomic analyses. Nat. Rev. Genet. 13, 227-232. doi: 10.1038/nrg3185

Wagner, A. (2005). Distributed robustness versus redundancy as causes of mutational robustness. Bioessays 27, 176-188. doi: 10.1002/bies.20170

Wang, P., Moore, B. M., Panchy, N. L., Meng, F., Lehti-Shiu, M. D., and Shiu, S.-H. (2018). Factors influencing gene family size variation among related species in a plant family, Solanaceae. Genome Biol. Evol. 10, 2596-2613. doi: 10.1093/gbe/ evy193

Wang, S., and Chen, Y. (2018). Phylogenomic analysis demonstrates a pattern of rare and long-lasting concerted evolution in prokaryotes. Commun. Biol. 1:12. doi: 10.1038/s42003-018-0014-x

Wang, S. S., and Adams, K. L. (2015). Duplicate gene divergence by changes in MicroRNA binding sites in Arabidopsis and Brassica. Genome Biol. Evol. 7 , 646-655. doi: 10.1093/gbe/evv023

Wang, Z., Sun, X. P., Zhao, Y., Guo, X. X., Jiang, H. F., Li, H. Y., et al. (2015). Evolution of gene regulation during transcription and translation. Genome Biol. Evol. 7, 1155-1167. doi: 10.1093/gbe/evv059

Wu, Z., Fang, D., Yang, R., Gao, F., An, X., Zhuo, X., et al. (2018). De novo genome assembly of Oryza granulata reveals rapid genome expansion and adaptive evolution. Commun. Biol. 1:84. doi: 10.1038/s42003-018-0089-4

Yang, Z. (2007). PAML 4: phylogenetic analysis by maximum likelihood. Mol. Biol. Evol. 24, 1586-1591. doi: 10.1093/molbev/msm088

Zhang, J. Z. (2003). Evolution by gene duplication: an update. Trends Ecol. Evol. 18, 292-298. doi: 10.1016/s0169-5347(03)00033-8

Zhang, J. Z., and He, X. L. (2005). Significant impact of protein dispensability on the instantaneous rate of protein evolution. Mol. Biol. Evol. 22, 1147-1155. doi: 10.1093/molbev/msi101

Zhao, D. Y., Hamilton, J. P., Hardigan, M., Yin, D. M., He, T., Vaillancourt, B., et al. (2017). Analysis of ribosome-associated mRNAs in rice reveals the importance of transcript size and GC content in translation. G3 7, 203-219. doi: $10.1534 / \mathrm{g} 3.116 .036020$

Zhong, Y., Karaletsos, T., Drewe, P., Sreedharan, V. T., Kuo, D., Singh, K., et al. (2017). RiboDiff: detecting changes of mRNA translation efficiency from ribosome footprints. Bioinformatics 33, 139-141. doi: 10.1093/bioinformatics/ btw585

Zou, C., Lehti-Shiu, M. D., Thomashow, M., and Shiu, S. H. (2009). Evolution of stress-regulated gene expression in duplicate genes of Arabidopsis thaliana. PLoS Genet. 5:e1000581. doi: 10.1371/journal.pgen.1000581

Conflict of Interest Statement: The authors declare that the research was conducted in the absence of any commercial or financial relationships that could be construed as a potential conflict of interest.

Copyright (c) 2019 Wang and Chen. This is an open-access article distributed under the terms of the Creative Commons Attribution License (CC BY). The use, distribution or reproduction in other forums is permitted, provided the original author(s) and the copyright owner(s) are credited and that the original publication in this journal is cited, in accordance with accepted academic practice. No use, distribution or reproduction is permitted which does not comply with these terms. 